\begin{tabular}{|c|c|c|c|c|c|}
\hline \multirow{2}{*}{$\begin{array}{l}\text { Number of } \\
\text { risk clusters } \\
\text { per } \\
\text { individuals }\end{array}$} & \multirow{2}{*}{$\begin{array}{l}\text { Number of } \\
\text { individuals (96) }\end{array}$} & \multicolumn{4}{|c|}{ RISK CCUSTER } \\
\hline & & $\begin{array}{c}\text { Systemic } \\
\text { autoimmunity }\end{array}$ & $\begin{array}{l}\text { Environmental } \\
\text { risk factors }\end{array}$ & $\begin{array}{c}\text { Genetic risk } \\
\text { factors }\end{array}$ & $\begin{array}{c}\text { Unclassifiled } \\
\text { Arthritis }\end{array}$ \\
\hline NONE & $8(7.15)$ & & & & \\
\hline \multirow{4}{*}{ ONE } & $19(16.858)$ & & & & \\
\hline & $10(2.85)$ & & & & \\
\hline & $2(1.85)$ & & & & \\
\hline & 0 & & & & \\
\hline \multirow{6}{*}{ Two } & 35 (31\%) & & & & \\
\hline & $9(856)$ & & & & \\
\hline & $5(4,48)$ & & & & \\
\hline & $2(1.85)$ & & & & \\
\hline & $\circ$ & & & & \\
\hline & 0 & & & & \\
\hline \multirow{4}{*}{ THREE } & $17(155)$ & & & & \\
\hline & $3(2.78)$ & & & & \\
\hline & 0 & & & & \\
\hline & 0 & & & & \\
\hline FouR & $2(2.88)$ & & & & \\
\hline
\end{tabular}

figure 1. Until now, 43 people were followed for 5 years; additional 52 telephone interviews were conducted. No evidence of RA (clinically or by history) was found. Conclusions: By now none of the followed individuals had any evidence of inflammatory joint disease based on patient-telephone interviews conducted and completed 5-year follow-up examinations. We were unable to find evidence for practical value of routine $A A B$ screening in healthy individuals without clinical signs of inflammatory joint diseases.

References:

[1] Gerlag et al. (2012) Ann Rheum Dis 2012;71:638-641.

Disclosure of Interest: None declared

DOI: 10.1136/annrheumdis-2017-eular.5951

\section{FRI0689 LIPID PEROXIDATION AS RISK FACTOR FOR ENDOTHELIAL DYSFUNCTION IN ANTIPHOSPHOLIPID SYNDROME (APS) PATIENTS}

L. Stojanovich, N. Stanisavljevic, A. Djokovic, M. Zdravkovic. Internal medicine department, University Hospital Center Bezanijska Kosa, Belgrade, Serbia

Background: APS pathophysiology is not clear enough yet since it has been implicated that aPL can activate cells (endothelial cells, monocytes, platelets), interfere with hemostatic reactions and activate complement reactions [1,2]

Objectives: The aim of this study was to evaluate oxidative stress markers and it relations to endothelial damage as risk factor for thrombosis in patients with primary (PAPS) and secondary (SAPS) antiphospholipid syndrome (APS) in correlation to traditional risk factors.

Methods: Flow mediated (FMD) and nitroglycerine (NMD) induced dilation of the brachial artery were studied in 140 APS patients (90 PAPS, 50 SAPS) and 40 controls matched by age, sex and conventional risk factors for atherosclerosis. Markers of oxidative stress: lipid hydroperoxydes ( $\mathrm{LOOH}$ ), advanced oxidation protein products (AOPP), total sulfhydryl grups (tSHG) and paraoxonase 1 activity (PON1) were determined by spectrophotometric method.

Results: Oxidative stress dominate in APS patients. $\mathrm{LOOH}$ and AOPP correlate to lipid fractions $(p<0.05)$, unlike PON1, tSHG that correlated to antiphospholipid antibody positivity $(p<0.05)$. FMD was lower in APS patients comparing to controls $(p<0.001)$. Cholesterol is independent variable for FMD impairment in control group ( $p=0.011) ; \mathrm{LOOH}$ in PAPS $(\mathrm{p}=0.004) ; \mathrm{LOOH}, \mathrm{aCL}$ and triglycerides in SAPS patients $(p=0.009, p=0.049$ and $p=0.012$, respectively).Combined predictive of $\mathrm{aCL}$ and $\mathrm{LOOH}$ is better for FMD impairment than $\mathrm{LOOH}$ alone in both PAPS and SAPS patients (AUC $0.727, \mathrm{p}=0.001,95 \% \mathrm{Cl} 0.616-0.837$ and AUC 0.824 , $\mathrm{p}<0.001,95 \% \mathrm{Cl}$ 0.690-0.957, repectively).

Conclusions: Endothelial dysfunction is doubtlessly present in APS patients with oxidative imbalance as additional risk factor among other risk factors for clinical event. Anticardiolipin antibodies affect endothelial dependent vasodilatation in SAPS patients. We demonstrated synergistic effect of aCL and $\mathrm{LOOH}$ as risk for endothelial impairment in both PAPS and SAPS patients.

\section{References:}

[1] Foltyn Zadura A, Memon AA, Stojanovich L, Perricone C, Conti F, Valesini G et al (2015) Factor H Autoantibodies in Patients with Antiphospholipid Syndrome and Thrombosis. J Rheumatol 42(10):1786-93.

[2] Stalc M, Poredos P, Peternel P, Tomsic M, Sebestjen M, Kveder T (2006) Endothelial function is impaired in patients with primary antiphospholipid syndrome. Thromb Res 118:455-461.

[3] Becarevic M, Stojanovich L, Ignjatovic S, Dopsaj V. The IgM isotype of antiannexin A5 antibodies and multiple positivity of conventional antiphospholipid antibodies: increasing the number of clinical manifestations of primary antiphospholipid syndrome. Clinical Rheumatology 03/2016; doi: 10.1007/ s10067-016-3230-0.
[4] Ames PR, Batuca JR, Ciampa A, lannaccone L, Delgado Alves J (2010) Clinical relevance of nitric oxide metabolites and nitrative stress in thrombotic primary antiphospholipid syndrome. J Rheumatol 37:2523-2530.

Acknowledgements: This work was supported by research grant number 175041, and TR 32040 for 2011 - 2017, issued by the Ministry of Science of the Republic of Serbia.

Disclosure of Interest: None declared

DOI: 10.1136/annrheumdis-2017-eular.2872

\section{FRI0690 RISK OF FRACTURE AMONG PATIENTS WITH GOUT: A POPULATION-BASED COHORT STUDY}

A. Abdul Sultan ${ }^{1}$, R. Whittle ${ }^{1}$, S. Muller ${ }^{1}$, E. Roddy ${ }^{1,2}$, C. Mallen $^{1}$, M. Blagojevic-Bucknall ${ }^{1}$, T. Helliwell ${ }^{1}$, S. Hider ${ }^{1,2}$, Z. Paskins $^{1,2},{ }^{1}$ Research Institute for Primary Care \& Health Sciences, Keele University, Staffordshire; ${ }^{2}$ Haywood Academic Rheumatology Centre, Staffordshire and Stoke-on-Trent Partnership Trust, Stoke-on-Trent, United Kingdom

Background: Gout is the most common type of inflammatory arthritis, affecting $2.4 \%$ of adults in the UK and is associated with a number of co-morbidities. Our understanding of the association between gout and fracture risk is limited with previous studies offering conflicting results.

Objectives: To determine the risk of fracture among gout patients and assess the potential impact of urate-lowering therapy (ULT) on fracture risk.

Methods: Utilising primary care records from Clinical Practice Research Datalink we identified patients with gout between 1990 and 2004 who were followed up until 2015. Each gout patient was individually matched to 5 individuals without gout based on age, sex, and registered practice. Absolute rate (AR) of fracture and hazard ratios (HR) were calculated using Cox regression models. We further stratified our analysis by age, gender and ULT prescription.

Results: We matched 35,857 patients with incident gout to 148,407 controls. Overall, we found no increased risk of fracture among gout patients compared to controls. However, men with no evidence of ULT had higher absolute risk of fracture compared to controls ( $A R=39$ versus 26 per 10,000 person-years) corresponding to a $23 \%(\mathrm{HR}=1.23 ; 95 \% \mathrm{Cl} 1.12-1.36)$ increased risk. The risk was particularly high for vertebral $(\mathrm{HR}=1.50 ; 95 \% \mathrm{Cl} 1.20-1.87)$ and wrist fracture $(\mathrm{HR}=1.45 ; 95 \% \mathrm{Cl} 1.21-1.74)$. Those treated with ULT had a 12\% (HR=0.88; $95 \% \mathrm{Cl}$ 0.79-0.98) lower risk of fracture. Similar findings were not observed for women.

Conclusions: We found higher risk of vertebral and wrist fractures among men with gout not prescribed ULT. Those prescribed ULT had lower risk of fracture compared to the general population. Further research is needed to understand the role of ULT in fracture prevention.

Acknowledgements: CDM is funded by the National Institute for Health Research (NIHR) Collaborations for Leadership in Applied Health Research and Care West Midlands, the NIHR School for Primary Care Research and a NIHR Research Professorship in General Practice. TH is funded by a NIHR Clinical Lectureship in General Practice and AAS is funded by NIHR Postdoctoral Fellowship. The views expressed are those of the author(s) and not necessarily those of the NHS, the NIHR or the Department of Health

Disclosure of Interest: None declared

DOI: 10.1136/annrheumdis-2017-eular.3761

\section{FRI0691 ASSOCIATION BETWEEN PERIODONTITIS AND THE RISK OF PALINDROMIC RHEUMATISM: A NATIONWIDE, POPULATION-BASED, CASE-CONTROL STUDY}

H.-H. Chen ${ }^{1}$, W.-C. Chao ${ }^{1}$, Y.-M. Chen ${ }^{1}$, D.-Y. Chen ${ }^{2} .{ }^{1}$ Department of Medical Research; ${ }^{2}$ Department of Internal Medicine, Taichung Veterans General Hospital, Taichung, Taiwan, Province of China

Background: Some patients with palindromic rheumatism (PR) may develop a chronic connective tissue disease, mainly rheumatoid arthritis (RA). About one to two-thirds of PR patients developed RA during a period of follow-up. Periodontitis (PD) has been found to be associated with RA risk. However, the association between PD and PR risk is unknown.

Objectives: To estimate the association between a history of PD and the risk of incident PR.

Methods: This study used a nationwide, administrative database to identify PR cases and non-PR controls. After exclusion of individuals with rheumatoid arthritis, systemic lupus erythematosus, Sjögren's syndrome, systemic sclerosis, dermatomyositis or polymyositis before the first PR diagnosis date (index date), we identified 4,421 newly-diagnosed PR cases from 2007 to 2012 and randomly selected 44,210 non-PR controls matched (1:10) for sex, age, and the year of the index date. After adjusting for comorbid diabetes mellitus, we estimated odds ratios (ORs) with $95 \%$ confidence intervals (Cls) by conditional logistic regression analysis to quantify the association between a history of PD and the risk of PR. The influences of the lag time and severity of PD were examined by calculating ORs for subgroups of patients based on time interval between the last PD-related visit and the index date and PD-related cumulative cost and visit number.

Results: This study showed an association between a history of PD and newly diagnosed PR $(\mathrm{OR}, 1.51 ; 95 \% \mathrm{Cl}, 1.41-1.61)$. The association remained significant after variation of PD definitions. The magnitude of the association was 